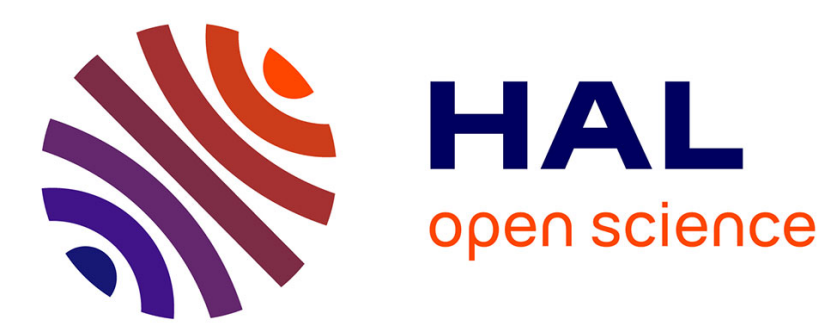

\title{
A deviation bound for $\alpha$-dependent sequences with applications to intermittent maps
}

\author{
J Dedecker, Florence Merlevède
}

\section{To cite this version:}

J Dedecker, Florence Merlevède. A deviation bound for $\alpha$-dependent sequences with applications to intermittent maps. Stochastics and Dynamics, 2017, 17 (1), 27pp. hal-01259827

\section{HAL Id: hal-01259827 \\ https://hal.science/hal-01259827}

Submitted on 21 Jan 2016

HAL is a multi-disciplinary open access archive for the deposit and dissemination of scientific research documents, whether they are published or not. The documents may come from teaching and research institutions in France or abroad, or from public or private research centers.
L'archive ouverte pluridisciplinaire HAL, est destinée au dépôt et à la diffusion de documents scientifiques de niveau recherche, publiés ou non, émanant des établissements d'enseignement et de recherche français ou étrangers, des laboratoires publics ou privés. 


\title{
A deviation bound for $\alpha$-dependent sequences with applications to intermittent maps
}

\author{
J. Dedecker ${ }^{a}$ and F. Merlevède ${ }^{b}$
}

${ }^{a}$ Université Paris Descartes, Sorbonne Paris Cité, Laboratoire MAP5 (UMR 8145).

Email: jerome.dedecker@parisdescartes.fr

${ }^{b}$ Université Paris Est, LAMA (UMR 8050), UPEM, CNRS, UPEC.

Email: florence.merlevede@u-pem.fr

Key words and phrases. Deviation inequalities, Moment inequalities, Stationary sequences, Invariance principles, Intermittency

Mathematical Subject Classification (2010). 60E15, 60G10, 60F17

\begin{abstract}
We prove a deviation bound for the maximum of partial sums of functions of $\alpha$-dependent sequences as defined in [2]. As a consequence, we extend the Rosenthal inequality of Rio [16] for $\alpha$-mixing sequences in the sense of Rosenblatt [18] to the larger class of $\alpha$-dependent sequences. Starting from the deviation inequality, we obtain upper bounds for large deviations and an Hölderian invariance principle for the Donsker line. We illustrate our results through the example of intermittent maps of the interval, which are not $\alpha$-mixing in the sense of Rosenblatt.
\end{abstract}

\section{Introduction}

For stationary $\alpha$-mixing sequences in the sense of Rosenblatt (see [18]) a Fuk-Nagaev type inequality has been proved by Rio (see Theorem 6.2 in [16]). This deviation inequality is very powerful and enables one to prove optimal results for the deviation of partial sums and a sharp Rosenthal type inequality (see Corollary 6.3 in [16]). The proof uses a blocking technique and the coupling property of $\alpha$-mixing coefficients.

Rio's inequality has been extended to a larger class of dependent sequences in [5]. In that paper, the authors noticed that one can use a dependency coefficient $\tau(n)$ whose definition is perfectly adapted to the coupling property, in the spirit of Rüschendorf [19]. The Fuk-Nagaev inequality for $\tau$-dependent sequences applies to many non-mixing sequences in the sense of Rosenblatt, such as contracting Markov chains or causal functions of infinite sequences of independent and identically distributed (i.i.d.) random variables.

However, although the property of $\tau$-dependency is much less restrictive than $\alpha$-mixing, it appears to be not well adapted to most of the usual dynamical systems. The main reason is that, to prove the Fuk-Nagaev inequality via blocking + coupling, one needs to control the dependency between the whole past and the whole future of the sequence. To the best of our knowledge, this can be done only for a very restricted class of dynamical systems (see Example 4 of Section 7.2 in [6]).

In the present paper, we prove a deviation bound for the maximum of partial sums of functions of stationary $\alpha$-dependent sequences as defined in [2]. More precisely, if $\mathbf{X}=\left(X_{i}\right)_{i \in \mathbb{Z}}$ is a strictly 
stationary sequence of real-valued random variables, our deviation inequality (see Proposition 5.1 ) is expressed in terms of a coefficient $\alpha_{2, \mathbf{X}}(n)$ which only controls the dependency between the past (before time 0) and any variable of the form $\mathbf{1}_{X_{i} \leq t, X_{j} \leq s}$ where $i, j \geq n$. Note that this coefficient can be exactly computed for the Markov chains associated to the intermittent maps introduced in [2] (see Subsection 4.1). We shall also describe precisely the class of observables $f\left(X_{i}\right)$ to which our results apply (in particular, this class contains the functions $f$ which are piecewise monotonic on open intervals with a finite number of branches, under an appropriate control of the tail of $f\left(X_{0}\right)$ ).

The proof of our deviation inequality still uses a blocking argument, but the coupling part is now replaced by a martingale approximation followed by an application of the Rosenthal-type inequality proved in [14]. The resulting inequality is not of the same kind as the usual FukNagaev inequality, but it seems to perform as well in most of the applications. For instance, it provides a full extension of the Rosenthal inequality of Rio [16] to the larger class of $\alpha$ dependent sequences (see our Theorem 3.1). We also use it to obtain upper bounds for large deviations and an Hölderian invariance principle for the Donsker line. Concerning the Hölderian invariance principle, we follow the approach of Giraudo [8], who recently obtained very precise results for mixing sequences ( $\alpha$-mixing in the sense of Rosenblatt, $\tau$-dependency and $\rho$-mixing) by applying sharp deviation inequalities for the maximum of partial sums.

The paper is organized as follows. In Section 2, we give the notations and definitions which will be used all along the paper. In Section 3, we present the main consequences of our deviation inequalities: moment bounds in Subsection 3.1, large deviation bounds in Subsection 3.2, Hölderian invariance principles in Subsection 3.3. The application of these results to intermittent maps are given in Section 4.1. In Section 5 our main deviation inequality is stated and proved. Finally, the proofs of the results of Section 3 are gathered in Section 6 .

\section{Definitions and notations}

Let us start with the notation $a_{n}(x) \ll b_{n}(x)$, which means that there exists a positive constant $C$ not depending on $n$ nor $x$ such that $a_{n}(x) \leq C b_{n}(x)$, for all positive integers $n$ and all real $x$.

\subsection{Stationary sequences and dependency coefficients}

Let $(\Omega, \mathcal{A}, \mathbb{P})$ be a probability space, and $T: \Omega \mapsto \Omega$ be a bijective bi-measurable transformation preserving the probability $\mathbb{P}$. Let $\mathcal{F}_{0}$ be a sub- $\sigma$-algebra of $\mathcal{A}$ satisfying $\mathcal{F}_{0} \subseteq T^{-1}\left(\mathcal{F}_{0}\right)$.

Let $Y_{0}$ be an $\mathcal{F}_{0}$-measurable and integrable real-valued random variable with distribution $P_{Y_{0}}$. Define the stationary sequence $\mathbf{Y}=\left(Y_{i}\right)_{i \in \mathbb{Z}}$ by $Y_{i}=Y_{0} \circ T^{i}$.

Let us now define the dependency coefficients of the sequence $\left(Y_{i}\right)_{i \in \mathbb{Z}}$ as in [2]. These coefficients are less restrictive than the usual mixing coefficients of Rosenblatt [18].

Definition 2.1. For any integrable random variable $Z$, let $Z^{(0)}=Z-\mathbb{E}(Z)$. For any random variable $V=\left(V_{1}, \cdots, V_{k}\right)$ with values in $\mathbb{R}^{k}$ and any $\sigma$-algebra $\mathcal{F}$, let

$$
\alpha(\mathcal{F}, V)=\sup _{\left(x_{1}, \ldots, x_{k}\right) \in \mathbb{R}^{k}}\left\|\mathbb{E}\left(\prod_{j=1}^{k}\left(\mathbf{1}_{V_{j} \leq x_{j}}\right)^{(0)} \mid \mathcal{F}\right)-\mathbb{E}\left(\prod_{j=1}^{k}\left(\mathbf{1}_{V_{j} \leq x_{j}}\right)^{(0)}\right)\right\|_{1} .
$$


For the stationary sequence $\mathbf{Y}=\left(Y_{i}\right)_{i \in \mathbb{Z}}$, let

$$
\alpha_{k, \mathbf{Y}}(0)=1 / 2 \text { and } \alpha_{k, \mathbf{Y}}(n)=\max _{1 \leq l \leq k} \sup _{n \leq i_{1} \leq \ldots \leq i_{l}} \alpha\left(\mathcal{F}_{0},\left(Y_{i_{1}}, \ldots, Y_{i_{l}}\right)\right) \text { for } n>0 .
$$

Note that $\alpha_{1, \mathbf{Y}}(n)$ is then simply given by

$$
\alpha_{1, \mathbf{Y}}(n)=\sup _{x \in \mathbb{R}}\left\|\mathbb{E}\left(\mathbf{1}_{Y_{n} \leq x} \mid \mathcal{F}_{0}\right)-F(x)\right\|_{1},
$$

where $F$ is the distribution function of $P_{Y_{0}}$.

All the results of the paper involve only the coefficients $\alpha_{1, \mathbf{X}}(n)$ and $\alpha_{2, \mathbf{X}}(n)$.

\subsection{Quantile functions and piecewise monotonic spaces}

In this subsection, we describe the functions spaces to which our results apply. Contrary to the usual mixing case, any function of a stationary $\alpha$-dependent sequence $\mathbf{Y}=\left(Y_{i}\right)_{i \in \mathbb{Z}}$ is not necessarily $\alpha$-dependent (meaning that its dependency coefficients do no necessarily tend to zero). Hence, we need to impose some constraints on the observables.

The first thing to notice is that, if $f$ is monotonic on some open interval and 0 elsewhere, and if $\mathbf{X}=\left(f\left(Y_{i}\right)\right)_{i \in \mathbb{Z}}$, then for any positive integer $k$,

$$
\alpha_{k, \mathbf{X}}(n) \leq 2^{k} \alpha_{k, \mathbf{Y}}(n) .
$$

As a consequence, if one can prove a deviation inequality for $\sum_{k=1}^{n} Y_{i}$ with an upper bound involving the coefficients $\left(\alpha_{k, \mathbf{Y}}(n)\right)_{n \geq 0}$ then it also holds for $\sum_{k=1}^{n} f\left(Y_{i}\right)$, where $f$ is monotonic on a single interval. In this case, the deviation inequality can be extended by linearity to convex combinations of such functions. Such classes are described in Definition 2.3 below.

First, we need a uniform control on the tail of our test functions by a given tail function $H$.

Definition 2.2. A function $H$ from $\mathbb{R}^{+}$to $[0,1]$ is a tail function if it is non-increasing, rightcontinuous and converges to zero at infinity. The quantile function $Q=H^{-1}$ is the generalized inverse of the tail function $H:$ for $u \in[0,1], Q(u)=\inf \{t \geq 0: H(t) \leq u\}$ (with the convention that $\inf \{\emptyset\}=\infty)$. For $p \geq 1$, we say that $Q$ belongs to $\mathbb{L}^{p}$ if $\int_{0}^{1} Q^{p}(u) d u<\infty$.

The function spaces are then defined as follows:

Definition 2.3. If $\mu$ is a probability measure on $\mathbb{R}$ and $Q=H^{-1}$ is an integrable quantile function, let $\widehat{\operatorname{Mon}}(Q, \mu)$ be the set of functions $g$ which are monotonic on some open interval of $\mathbb{R}$ and null elsewhere and such that $\mu(|g|>t) \leq H(t)$ for any $t \in \mathbb{R}^{+}$. Let $\widetilde{\mathcal{F}}(Q, \mu)$ be the closure in $\mathbb{L}^{1}(\mu)$ of the set of functions which can be written as $\sum_{\ell=1}^{L} a_{\ell} f_{\ell}$, where $\sum_{\ell=1}^{L}\left|a_{\ell}\right| \leq 1$ and $f_{\ell}$ belongs to $\widetilde{\operatorname{Mon}}(Q, \mu)$.

Note that a function belonging to $\widetilde{\mathcal{F}}(Q, \mu)$ is allowed to blow up at an infinite number of points. Note also that any function $f$ with bounded variation (BV) such that $|f| \leq M_{1}$ and $\|d f\| \leq M_{2}$ belongs to the class $\widetilde{\mathcal{F}}(Q, \mu)$ for any $\mu$ and the quantile function $Q \equiv M_{1}+2 M_{2}$ (here $\|d f\|$ denotes the variation norm of the signed measure $d f$ ). Moreover, if a function $f$ is piecewise monotonic with $N$ branches, then it belongs to $\widetilde{\mathcal{F}}(Q, \mu)$ for the quantile function $Q=H^{-1}$ and $H(t)=\mu(|f|>t / N)$. Finally, let us emphasize that there is no requirement on the modulus of continuity for functions in $\widetilde{\mathcal{F}}(Q, \mu)$. 


\section{Main results for $\alpha$-dependent sequences}

In Proposition 5.1 given in Section 5, we give a general deviation inequality for $\alpha$-dependent sequences. In this section, we present some striking applications of this inequality.

We shall use the following notations: for $u \in[0,1]$ and $k \in \mathbb{N}^{*}$, let

$$
\alpha_{k, \mathbf{Y}}^{-1}(u)=\min \left\{q \in \mathbb{N}: \alpha_{k, \mathbf{Y}}(q) \leq u\right\}=\sum_{n=0}^{\infty} \mathbf{1}_{u<\alpha_{k}, \mathbf{Y}}(n) .
$$

Note that $\alpha_{1, \mathbf{Y}}(n) \leq \alpha_{2, \mathbf{Y}}(n)$, and consequently $\alpha_{1, \mathbf{Y}}^{-1} \leq \alpha_{2, \mathbf{Y}}^{-1}$.

\subsection{Moment inequalities}

Our first result is the following Rosenthal-type inequality for the maximum of partial sums of $\alpha$-dependent sequences for all powers $p \geq 2$.

Theorem 3.1. Let $p \geq 2$ and let $Q$ be a quantile function in $\mathbb{L}^{p}$. Let $\mathbf{Y}=\left(Y_{i}\right)_{i \in \mathbb{Z}}$, where $Y_{i}=Y_{0} \circ T^{i}$. Denote by $P_{Y_{0}}$ the distribution of $Y_{0}$. Let $X_{i}=f\left(Y_{i}\right)-\mathbb{E}\left(f\left(Y_{i}\right)\right)$, where $f$ belongs to $\widetilde{\mathcal{F}}\left(Q, P_{Y_{0}}\right)$ and let $S_{n}=\sum_{k=1}^{n} X_{k}$. Then

$$
\left\|\max _{1 \leq k \leq n}\left|S_{k}\right|\right\|_{p}^{p} \ll n^{p / 2}\left(\int_{0}^{1}\left(\alpha_{1, \mathbf{Y}}^{-1}(u) \wedge n\right) Q^{2}(u) d u\right)^{p / 2}+n \int_{0}^{1}\left(\alpha_{2, \mathbf{Y}}^{-1}(u) \wedge n\right)^{p-1} Q^{p}(u) d u .
$$

Remark 3.1. Note that Inequality (3.2) can be written as follows:

$$
\left\|\max _{1 \leq k \leq n}\left|S_{k}\right|\right\|_{p}^{p} \ll n^{p / 2}\left(\sum_{k=0}^{n-1} \int_{0}^{\alpha_{1, \mathbf{Y}}(k)} Q^{2}(u) d u\right)^{p / 2}+n \sum_{k=0}^{n-1}(k+1)^{p-2} \int_{0}^{\alpha_{2, \mathbf{Y}}(k)} Q^{p}(u) d u .
$$

Remark 3.2. Inequality (3.2) is an extension of Rio's inequality for $\alpha$-mixing sequences (see Theorem 6.3 in [16]) to the larger class of $\alpha$-dependent sequences as defined in (2.1) (with $k=2$ for the index of the dependency). Note that Rio's inequality cannot be applied to the class of GPM maps described in Subsection 4.1, because the associated Markov chains of such maps are not $\alpha$-mixing in the sense of Rosenblatt. Note also that Inequality (3.3) implies in particular that if $p \geq 2$ and

$$
\sum_{k \geq 0}(k+1)^{p / 2-1} \int_{0}^{\alpha_{2, \mathbf{Y}}(k)} Q^{p}(u) d u<\infty, \text { then }\left\|\max _{1 \leq k \leq n}\left|S_{k}\right|\right\|_{p}^{p} \ll n^{p / 2} .
$$

We refer to Section 6.4 in Rio [17] for other possible consequences of Inequality (3.2) (see in particular Corollary 6.1 in Rio [17]).

\subsection{Large deviation inequalities}

In this section, we give some upper bounds for the quantity

$$
\mathbb{P}\left(\frac{1}{n} \max _{1 \leq k \leq n}\left|S_{k}\right| \geq x\right) .
$$

We shall use the notation

$$
R(u)=\alpha_{2, \mathbf{Y}}^{-1}(u) Q(u), \quad \text { for } u \in[0,1] .
$$


Theorem 3.2. Let $Q$ be a quantile function in $\mathbb{L}^{1}$, and let $Y_{i}, f, X_{i}$ and $S_{n}$ be as in Theorem 3.1.

1. Assume that, for $p \geq 2$,

$$
\sup _{x>0} x^{p-1} \int_{0}^{1} Q(u) \mathbf{1}_{R(u)>x} d u<\infty .
$$

Then, for $p>2$, any $a \in(p-1, p)$ and any $x>0$,

$$
\mathbb{P}\left(\frac{1}{n} \max _{1 \leq k \leq n}\left|S_{k}\right| \geq x\right) \ll \frac{1}{n^{a} x^{2 a}}+\frac{1}{n^{p-1} x^{p}} .
$$

For $p=2$, any $a \in(1,2)$, any $c \in(0,1)$ and any $x>0$,

$$
\mathbb{P}\left(\frac{1}{n} \max _{1 \leq k \leq n}\left|S_{k}\right| \geq x\right) \ll \frac{1}{n^{a c} x^{a(1+c)}}+\frac{1}{n x^{2}} .
$$

2. Assume that, for $p \geq 2$,

$$
\int_{0}^{1}\left(\alpha_{2, \mathbf{Y}}^{-1}(u)\right)^{p-1} Q^{p}(u) d u<\infty
$$

Then, for any $a \in(p-1, p)$ and any $x>0$,

$$
\sum_{n>0} n^{p-2} \mathbb{P}\left(\frac{1}{n} \max _{1 \leq k \leq n}\left|S_{k}\right| \geq x\right) \ll \frac{1}{x^{2 a}}+\frac{1}{x^{p}} .
$$

Remark 3.3. We consider here the case where $p \in(1,2)$. Let $Q$ be a quantile function in $\mathbb{L}^{1}$, and let $Y_{i}, f, X_{i}$ and $S_{n}$ be as in Theorem 3.1. Following the proof of Theorem 3.2 and using Proposition 1 in [3] instead of Inequality (5.1), one can prove that:

1. If (3.5) holds, then for any $x>0$,

$$
\mathbb{P}\left(\frac{1}{n} \max _{1 \leq k \leq n}\left|S_{k}\right| \geq x\right) \ll \frac{1}{n^{p-1} x^{p}} .
$$

2. If (3.8) holds, then for any $x>0$,

$$
\sum_{n>0} n^{p-2} \mathbb{P}\left(\frac{1}{n} \max _{1 \leq k \leq n}\left|S_{k}\right| \geq x\right) \ll \frac{1}{x^{p}}
$$

We refer to Subsection 4.2 in [2] to see how to apply Proposition 1 in [3] to $\alpha$-dependent sequences. Note that in the case $p \in(1,2)$, the conditions (3.5) and (3.8) can be slightly weakened by replacing $\alpha_{2, \mathbf{Y}}^{-1}(u)$ by $\alpha_{1, \mathbf{Y}}^{-1}(u)$ in the definition of $R(u)$.

Remark 3.4. Since $x^{p-1} \mathbf{1}_{R(u)>x} \leq(R(u))^{p-1}$, we see that the condition (3.8) is stronger than (3.5). Note also that (3.8) is equivalent to

$$
\sum_{k=0}^{\infty}(k+1)^{p-2} \int_{0}^{\alpha_{2, \mathbf{Y}}(k)} Q^{p}(u) d u<\infty .
$$

From (3.6), (3.7) and (3.10), it follows that, for any $x>0$ and any $p>1$,

$$
\mathbb{P}\left(\max _{1 \leq k \leq n}\left|S_{k}\right| \geq n x\right)=O\left(\frac{1}{n^{p-1}}\right) .
$$

From (3.9) and (3.11), it follows that, for any $x>0$ and any $p>1$,

$$
\mathbb{P}\left(\max _{1 \leq k \leq n}\left|S_{k}\right| \geq n x\right)=o\left(\frac{1}{n^{p-1}}\right) .
$$


Remark 3.5. Let $m$ be a non-negative integer. As usual, the stationary sequence $\mathbf{X}$ is $m$ dependent if $\sigma\left(X_{i}, i \leq 0\right)$ is independent of $\sigma\left(X_{i}, i \geq m+1\right)$, and $m=0$ corresponds to the case of i.i.d random variables. If $\mathbf{X}$ is a stationary $m$-dependent sequence of centered random variables, we infer from Theorem 3.2 (resp. Remark 3.3) that (3.6)-(3.7) (resp. (3.10)) holds for $p \geq 2$ (resp. for $p \in(1,2)$ ) as soon as

$$
\sup _{x>0} x^{p-1} \mathbb{E}\left(\left|X_{0}\right| \mathbf{1}_{\left|X_{0}\right|>x}\right)<\infty .
$$

Since $p>1$, It is easy to see that (3.13) is equivalent to

$$
\sup _{x>0} x^{p} \mathbb{P}\left(\left|X_{0}\right|>x\right)<\infty,
$$

meaning that $X_{0}$ has a weak moment of order $p$. In the same way, (3.9) (resp. (3.11)) holds for $p \geq 2$ (resp. for $p \in(1,2)$ ) as soon as $\mathbb{E}\left(\left|X_{0}\right|^{p}\right)<\infty$. In particular, if $\mathbb{E}\left(\left|X_{0}\right|^{p}\right)<\infty$ for $p>1$, then (3.12) holds. Now, according to Proposition 2.6 in [11], the estimate (3.12) cannot be essentially improved in the i.i.d. case.

\subsection{Hölderian invariance principles}

Let $Y_{i}, f, X_{i}$ and $S_{n}$ be as in Theorem 3.1, and define

$$
W_{n}(t)=\frac{1}{\sqrt{n}} S_{[n t]}+\frac{(n t-[n t])}{\sqrt{n}} X_{[n t]+1} .
$$

From [2] we know that, if

$$
\int_{0}^{1} \alpha_{2, \mathbf{Y}}^{-1}(u) Q^{2}(u) d u<\infty,
$$

then the process $\left\{W_{n}(t), t \in[0,1]\right\}$ converges in distribution in the space $C\left([0,1],\|\cdot\|_{\infty}\right)$ of continuous bounded function on $[0,1]$ to $\sigma W$, where $W$ is a standard Brownian motion and

$$
\sigma^{2}=\operatorname{Var}\left(X_{0}\right)+2 \sum_{k>0} \operatorname{Cov}\left(X_{0}, X_{k}\right) .
$$

For $\beta \in(0,1]$, let $\mathcal{H}_{\beta}([0,1])$ be the Banach space of $\beta$-Hölder functions from $[0,1] \rightarrow \mathbb{R}$, endowed with the norm

$$
|f|_{\beta}=|f(0)|+w_{\beta}(f, 1)
$$

where

$$
w_{\beta}(f, x)=\sup _{s, t \in[0,1],|t-s| \leq x} \frac{|f(t)-f(s)|}{|t-s|^{\beta}} .
$$

We denote by $\mathcal{H}_{\beta}^{0}([0,1])$ the subspace of $\mathcal{H}_{\beta}([0,1])$ of all functions $f$ such that $\lim _{x \rightarrow 0} w_{\beta}(f, x)=$ 0 . It is well known (see [1]) that $\mathcal{H}_{\beta}^{0}([0,1])$ is a closed subspace of $\mathcal{H}_{\beta}([0,1])$, so that it is a Banach space (for the norm $|\cdot|_{\beta}$ ), and that it is separable (whereas $\mathcal{H}_{\beta}([0,1]$ ) is not).

Since the sample paths of the Brownian motion are $\beta$-Hölder continuous for any $\beta<1 / 2$, we may consider $W$ as a random variable taking values in $\mathcal{H}_{\beta}^{0}([0,1]), \beta<1 / 2$. It is therefore natural to look for sufficient conditions ensuring that the convergence of $\left\{W_{n}(t), t \in[0,1]\right\}$ to $\sigma W$ takes place in the space $\mathcal{H}_{\beta}^{0}([0,1])$.

In the case of strong mixing sequences in the sense of Rosenblatt, Giraudo [8] recently proved such an invariance principle under a sharp condition expressed in terms of moments of the random variables and strong mixing rates. As we shall see, Giraudo's result can be extended to $\alpha$-dependent sequences. 
Theorem 3.3. Let $Q$ be an integrable quantile function, and let $Y_{i}, f, X_{i}$ and $S_{n}$ be as in Theorem 3.1. Assume that, for $p>2$,

$$
\lim _{x \rightarrow \infty} x^{p-1} \int_{0}^{1} Q(u) \mathbf{1}_{R(u)>x} d u=0
$$

where $R$ is defined in (3.4). Let $\delta=(1 / 2)-(1 / p)$. Then the process $\left\{W_{n}(t), t \in[0,1]\right\}$ converges in distribution in $\mathcal{H}_{\delta}^{0}([0,1])$ to $\sigma W$, where $W$ is a standard Brownian motion and $\sigma^{2}$ is defined in (3.15).

Remark 3.6. In his paper [8], Giraudo obtains also sharp results for $\tau$-dependent and $\rho$-mixing sequences. In a second paper [9], he also proves Hölderian invariance principles for other classes of stationary sequences via martingale approximations.

Remark 3.7. Applying Markov's inequality at order $p-1$, we see that the condition (3.8) implies (3.16). In the stationary $m$-dependent case, we infer from Theorem 3.3 that the process $\left\{W_{n}(t), t \in[0,1]\right\}$ converges in distribution in $\mathcal{H}_{\delta}^{0}([0,1])$ to $\sigma W$ as soon as

$$
\lim _{x \rightarrow \infty} x^{p-1} \mathbb{E}\left(\left|X_{0}\right| \mathbf{1}_{\left|X_{0}\right|>x}\right)=0 .
$$

Since $p>1$, It is easy to see (see Remark 2.3 in [8]) that (3.17) is equivalent to

$$
\lim _{x \rightarrow \infty} x^{p} \mathbb{P}\left(\left|X_{0}\right|>x\right)=0 .
$$

Note that, in the i.i.d. case, the condition (3.18) is necessary and sufficient for the invariance principle in $\mathcal{H}_{\delta}^{0}([0,1])$ (see $\left.[15]\right)$.

\section{Application to intermittent maps}

\subsection{Intermittent maps}

Let us first recall the definition of the generalized Pomeau-Manneville maps introduced in [2].

Definition 4.1. A map $\theta:[0,1] \rightarrow[0,1]$ is a generalized Pomeau-Manneville map (or GPM map) of parameter $\gamma \in(0,1)$ if there exist $0=y_{0}<y_{1}<\cdots<y_{d}=1$ such that, writing $I_{k}=\left(y_{k}, y_{k+1}\right)$,

1. The restriction of $\theta$ to $I_{k}$ admits a $C^{1}$ extension $\theta_{(k)}$ to $\overline{I_{k}}$.

2. For $k \geq 1, \theta_{(k)}$ is $C^{2}$ on $\overline{I_{k}}$, and $\left|\theta_{(k)}^{\prime}\right|>1$.

3. $\theta_{(0)}$ is $C^{2}$ on $\left(0, y_{1}\right]$, with $\theta_{(0)}^{\prime}(x)>1$ for $x \in\left(0, y_{1}\right], \theta_{(0)}^{\prime}(0)=1$ and $\theta_{(0)}^{\prime \prime}(x) \sim c x^{\gamma-1}$ when $x \rightarrow 0$, for some $c>0$.

4. $\theta$ is topologically transitive.

The third condition ensures that 0 is a neutral fixed point of $\theta$, with $\theta(x)=x+c^{\prime} x^{1+\gamma}(1+o(1))$ when $x \rightarrow 0$. The fourth condition is necessary to avoid situations where there are several absolutely continuous invariant measures, or where the neutral fixed point does not belong to the support of the absolutely continuous invariant measure. 


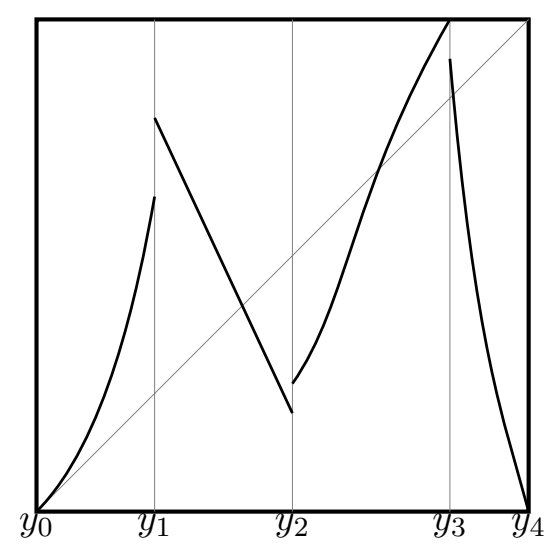

Figure 1: The graph of a GPM map, with $d=4$

The following well known example of GPM map with only two branches has been introduced by Liverani, Saussol and Vaienti [12]:

$$
\theta(x)= \begin{cases}x\left(1+2^{\gamma} x^{\gamma}\right) & \text { if } x \in[0,1 / 2[ \\ 2 x-1 & \text { if } x \in[1 / 2,1]\end{cases}
$$

As quoted in [2], a GPM map $\theta$ admits a unique invariant absolutely continuous (with respect to the Lebesgue measure) probability $\nu$ with density $h$. Moreover, it is ergodic, has full support, and $x^{\gamma} h(x)$ is bounded above and below by positive constants.

We shall apply the results of Sections 3.1, 3.2 and 3.3 to the partial sums

$$
S_{n}(f)=\sum_{k=1}\left(f \circ \theta^{k}-\nu(f)\right)
$$

where $\theta$ is a GPM map, and $f$ belongs to the space $\widetilde{\mathcal{F}}(Q, \nu)$ for some integrable quantile function $Q$.

To do this, we shall make use of the results of [2]. It is proved in that paper that there exists a stationary Markov chain $\mathbf{Y}=\left(Y_{i}\right)_{i \in \mathbb{Z}}$ such that, on the probability space $([0,1], \nu)$, the random vector $\left(\theta, \theta^{2}, \ldots, \theta^{n}\right)$ is distributed as $\left(Y_{n}, Y_{n-1}, \ldots, Y_{1}\right)$.

In particular, on $([0,1], \nu)$, the partial sum $S_{n}(f)$ defined in (4.2) is distributed as $\sum_{k=1}^{n} X_{i}$ with $X_{i}=f\left(Y_{i}\right)-\nu(f)$. Moreover, since

$$
\max _{1 \leq k \leq n}\left|S_{k}(f)\right| \text { is distributed as } \max _{1 \leq k \leq n}\left|\sum_{i=k}^{n} X_{i}\right|,
$$

we easily derive that, for any $x \geq 0$,

$$
\nu\left(\max _{1 \leq k \leq n}\left|S_{k}(f)\right| \geq x\right) \leq \mathbb{P}\left(2 \max _{1 \leq k \leq n}\left|\sum_{k=1}^{n} X_{i}\right| \geq x\right) .
$$

From Proposition 1.17 (and the comments right after) in [2], we know that for any positive integer $k$, there exist two positive constants $C$ and $D$ such that, for any $n>0$,

$$
\frac{D}{n^{(1-\gamma) / \gamma}} \leq \alpha_{k, \mathbf{Y}}(n) \leq \frac{C}{n^{(1-\gamma) / \gamma}}
$$


This control of the coefficients $\alpha_{k, \mathbf{Y}}(n)$ (for $k=2$ ), together with Inequality (4.3) and the control $\nu(|f|>t) \leq H(t)$, are all we need to apply the results of Sections 3.1, 3.2 and 3.3 to the partial sums $S_{n}(f)$.

\subsection{Moment bounds for intermittent maps}

In this subsection, we shall prove moment inequalities for $\max _{1 \leq k \leq n}\left|S_{k}(f)\right|$ when $f \in \widetilde{\mathcal{F}}(Q, \nu)$ and $Q(u) \ll u^{-b}$ for $b \in[0,1)$.

In particular, our results apply to the two simple examples:

1. If $f$ is positive and non increasing on $(0,1)$, with

$$
f(x) \leq \frac{C}{x^{s}} \quad \text { near } 0, \text { for some } C>0 \text { and } s \in[0,1-\gamma),
$$

then $f$ belongs to $\widetilde{\mathcal{F}}(Q, \nu)$ with $Q(u) \ll u^{-s /(1-\gamma)}$ (meaning that $b=s /(1-\gamma)$ ).

2. If $f$ is positive and non increasing on $(0,1)$, with

$$
f(x) \leq \frac{C}{(1-x)^{s}} \quad \text { near } 1, \text { for some } C>0 \text { and } s \in[0,1)
$$

then $f$ belongs to $\widetilde{\mathcal{F}}(Q, \nu)$ with $Q(u) \ll u^{-s}$ (meaning that $b=s$ ).

- Assume first that $p>2$ and $b \in[0,1 / p)$ in such a way that $\int_{0}^{1} Q^{p}(u) d u<\infty$ for $p>2$. From (4.3) and Theorem 3.1, we infer that, for any $f$ in $\widetilde{\mathcal{F}}(Q, \nu)$,

$$
\left\|\max _{1 \leq k \leq n}\left|S_{k}(f)\right|\right\|_{p}^{p} \ll \begin{cases}n^{p / 2} & \text { if } b \leq(2-\gamma(p+2)) /(2 p(1-\gamma)) \\ n^{(p \gamma+(\gamma-1)(1-p b)) / \gamma} & \text { if } b>(2-\gamma(p+2)) /(2 p(1-\gamma)) .\end{cases}
$$

- Assume now that $p=2$ and $b \in[0,1 / 2)$ in such a way that $\int_{0}^{1} Q^{2}(u) d u<\infty$. From (4.3) and Theorem 3.1, we infer that, for any $f$ in $\widetilde{\mathcal{F}}(Q, \nu)$,

$$
\left\|\max _{1 \leq k \leq n}\left|S_{k}(f)\right|\right\|_{2}^{2} \ll \begin{cases}n & \text { if } b<(1-2 \gamma) /(2(1-\gamma)) \\ n \ln (n) & \text { if } b=(1-2 \gamma) /(2(1-\gamma)) \\ n^{(2 \gamma+(\gamma-1)(1-2 b)) / \gamma} & \text { if } b>(1-2 \gamma) /(2(1-\gamma)) .\end{cases}
$$

- Assume now that $p \in(1,2)$ and $b \in[0,1 / p)$ in such a way that $\int_{0}^{1} Q^{p}(u) d u<\infty$ for $p<2$. Applying Remark 8 in [3] (see section 4.2 in [2] for its application to $\alpha$-dependent sequences) the following upper bounds hold.

$$
\left\|\max _{1 \leq k \leq n}\left|S_{k}(f)\right|\right\|_{p}^{p} \ll \begin{cases}n & \text { if } b<(1-p \gamma) /(p(1-\gamma)) \\ n \ln (n) & \text { if } b=(1-p \gamma) /(p(1-\gamma)) \\ n^{(p \gamma+(\gamma-1)(1-p b)) / \gamma} & \text { if } b>(1-p \gamma) /(p(1-\gamma)) .\end{cases}
$$

Moreover, if $b=(1-p \gamma) /(p(1-\gamma))$, Proposition 1 in [3] gives the upper bound

$$
\nu\left(\max _{1 \leq k \leq n}\left|S_{k}(f)\right| \geq x\right) \ll \frac{n}{x^{p}} .
$$


Remark 4.2. When $f$ is a bounded variation function (which corresponds to the case $b=0$ ) all the rates above are given in Theorem 4.5 of [4], and these rates are optimal (see the discussion in Section 4.4 of [4]).

Remark 4.3. Let us consider the case where $f$ satisfies (4.4) (in which case $b=s /(1-\gamma)$ ).

In that case, if $s=(1-2 \gamma) / 2$, the upper bound (4.7) gives that

$$
\left\|\max _{1 \leq k \leq n}\left|S_{k}(f)\right|\right\|_{2}^{2} \ll n \ln (n) .
$$

This upper bound is in accordance with a result by Gouëzel [10]. He proved that, if $f$ is exactly of the form $f(x)=x^{-(1-2 \gamma) / 2}$ and $\theta$ is the LSV map defined by $(4.1)$, then $S_{n}(f) / \sqrt{n \ln (n)}$ converges in distribution to a non-degenerate Gaussian random variable.

Now, if $s=(1-p \gamma) / p$, the upper bound (4.8) holds. This is also in accordance with a result by Gouëzel [10]. He proved that, if $f$ is exactly of the form $f(x)=x^{-(1-p \gamma) / p}$ and $\theta$ is the LSV map defined by (4.1), then for any positive real $x$,

$$
\lim _{n \rightarrow \infty} \nu\left(\frac{1}{n^{1 / p}}\left|S_{n}(f)\right|>x\right)=\mathbb{P}\left(\left|Z_{p}\right|>x\right),
$$

where $Z_{p}$ is a $p$-stable random variable such that $\lim _{x \rightarrow \infty} x^{p} \mathbb{P}\left(\left|Z_{p}\right|>x\right)=c>0$.

\subsection{Large deviations for intermittent maps}

Let $f$ in $\widetilde{\mathcal{F}}(Q, \nu)$ with $Q(u) \ll u^{-b}$ for some $b \in[0,1)$.

- Assume first that $\gamma+b(1-\gamma)<1 / 2$, and let $p=1 /(\gamma+b(1-\gamma))$. Applying Inequality (3.6), we get that, for any $a \in(p-1, p)$ and any $x>0$,

$$
\nu\left(\frac{1}{n} \max _{1 \leq k \leq n}\left|S_{k}(f)\right| \geq x\right) \ll \frac{1}{n^{a} x^{2 a}}+\frac{1}{n^{p-1} x^{p}} .
$$

- Assume now that $\gamma+b(1-\gamma)=1 / 2$. Applying Inequality (3.7) we get that, for any $a \in(1,2)$, any $c \in(0,1)$ and any $x>0$,

$$
\nu\left(\frac{1}{n} \max _{1 \leq k \leq n}\left|S_{k}(f)\right| \geq x\right) \ll \frac{1}{n^{a c} x^{a(1+c)}}+\frac{1}{n x^{2}} .
$$

- Assume now that $\gamma+b(1-\gamma) \in(1 / 2,1)$, and let $p=1 /(\gamma+b(1-\gamma))$. Applying (4.8), we get that, for any $x>0$,

$$
\nu\left(\frac{1}{n} \max _{1 \leq k \leq n}\left|S_{k}(f)\right| \geq x\right) \ll \frac{1}{n^{p-1} x^{p}} .
$$

Remark 4.4. Let $b \in[0,1)$ and $p=1 /(\gamma+b(1-\gamma))$. From the preceeding upper bounds, we infer that there exists a function $f_{b, \gamma}$ from $\mathbb{R}^{+}$to $\mathbb{R}^{+}$such that for any $x>0$,

$$
\nu\left(\frac{1}{n} \max _{1 \leq k \leq n}\left|S_{k}(f)\right| \geq x\right) \ll \frac{f_{b, \gamma}(x)}{n^{p-1}} .
$$

Moreover $\sup _{x>\varepsilon} x^{p} f_{b, \gamma}(x)<\infty$ for any $\varepsilon>0$. 
Remark 4.5. When $f$ is a bounded variation function (which corresponds to the case $b=0$ ), we obtain from (4.9) that, for any $x>0$,

$$
\nu\left(\frac{1}{n} \max _{1 \leq k \leq n}\left|S_{k}(f)\right| \geq x\right) \ll \frac{f_{0, \gamma}(x)}{n^{(1-\gamma) / \gamma}} .
$$

Note that the upper bound (4.10) (with $S_{n}(f)$ instead of the maximum) has been already obtained by Melbourne ([13], Example 1.6) when $\theta$ is the LSV map defined by (4.1) and $f$ is Hölder continuous (as a consequence of a very general result on slowly mixing dynamical system). In that case, the bound is optimal (see again Example 1.6 in [13]).

\subsection{Hölderian invariance principles for intermittent maps}

Assume now that $f$ belongs to $\widetilde{\mathcal{F}}(Q, \nu)$ for some $Q$ such that $Q(u) \leq u^{-b} \varepsilon(u)$, where $b \in(0,1)$ and $\varepsilon$ is a bounded function such that $\varepsilon(u) \rightarrow 0$ as $u \rightarrow 0$.

In particular, our results apply to the two simple examples:

1. If $f$ is positive and non increasing on $(0,1)$, with

$$
f(x) \leq \frac{\varepsilon(x)}{x^{s}} \quad \text { near } 0, \text { for some } s \in[0,1-\gamma),
$$

then the assumption on $Q$ is satisfied with $b=s /(1-\gamma)$.

2. If $f$ is positive and non increasing on $(0,1)$, with

$$
f(x) \leq \frac{\varepsilon(1-x)}{(1-x)^{s}} \quad \text { near } 1, \text { for some } s \in[0,1)
$$

then the assumption on $Q$ is satisfied with $b=s$.

Let then

$$
W_{n}(f, t)=\frac{1}{\sqrt{n}} S_{[n t]}(f)+\frac{(n t-[n t])}{\sqrt{n}} f \circ \theta^{[n t]+1} .
$$

Assume that $\gamma+b(1-\gamma)<1 / 2$, and let $\delta=(1 / 2)-\gamma-b(1-\gamma)$. Applying Theorem 3.3, we infer that, on the probabilty space $([0,1], \nu)$, the process $\left\{W_{n}(f, t), t \in[0,1]\right\}$ converges in distribution in $\mathcal{H}_{\delta}^{0}([0,1])$ to $\sigma(f) W$, where $W$ is a standard Brownian motion and

$$
\sigma^{2}(f)=\nu\left((f-\nu(f))^{2}\right)+2 \sum_{k>0} \nu\left((f-\nu(f)) \cdot f \circ T^{k}\right) .
$$

Remark 4.6. When $f$ is a bounded variation function and $\gamma<1 / 2$, we infer that the process $\left\{W_{n}(f, t), t \in[0,1]\right\}$ converges in distribution in $\mathcal{H}_{\delta}^{0}([0,1])$ to $\sigma(f) W$, for any $\delta<1 / 2-\gamma$.

\section{A deviation inequality for the maximum of partial sums}

Recall first that the functions $\alpha_{1, \mathbf{Y}}^{-1}$ and $\alpha_{2, \mathbf{Y}}^{-1}$ have been defined in (3.1). Our key deviation inequality for $\alpha$-dependent sequences is given below. 
Proposition 5.1. Let $\mathbf{Y}=\left(Y_{i}\right)_{i \in \mathbb{Z}}$, where $Y_{i}=Y_{0} \circ T^{i}$. Denote by $P_{Y_{0}}$ the distribution of $Y_{0}$, and let $Q$ be a quantile function in $\mathbb{L}^{1}$. Let $X_{i}=f\left(Y_{i}\right)-\mathbb{E}\left(f\left(Y_{i}\right)\right)$, where $f$ belongs to $\widetilde{\mathcal{F}}\left(Q, P_{Y_{0}}\right)$. Given a positive integer $n$, define

$$
R_{n}(u)=\left(\alpha_{2, \mathbf{Y}}^{-1}(u) \wedge n\right) Q(u), \quad \text { for } u \in[0,1]
$$

and

$$
L_{n}(x)=R_{n}^{-1}(x)=\inf \left\{u \in[0,1]: R_{n}(u) \leq x\right\}, \quad \text { for } x \geq 0 .
$$

Let $S_{n}=\sum_{k=1}^{n} X_{k}$. For any $\left.x>0, r>2, \beta \in\right] r-2, r[$ and

$$
s_{n}^{2}(x)=n \int_{L_{n}(x)}^{1}\left(\alpha_{1, \mathbf{Y}}^{-1}(u) \wedge n\right) Q^{2}(u) d u,
$$

the following deviation bound holds

$$
\begin{aligned}
\mathbb{P}\left(\max _{1 \leq k \leq n}\left|S_{k}\right| \geq x\right) \ll \frac{s_{n}^{r}(x)}{x^{r}}+\frac{n}{x} \int_{0}^{L_{n}(x)} Q(u) d u+ & \frac{n}{x^{1+\beta / 2}} \int_{0}^{L_{n}(x)} R_{n}^{\beta / 2}(u) Q(u) d u \\
& +\frac{n}{x^{1+r / 2}} \int_{L_{n}(x)}^{1} R_{n}^{r / 2}(u) Q(u) d u .
\end{aligned}
$$

Remark 5.2. The quantity $s_{n}^{2}(x)$ can be bounded as follows:

$$
s_{n}^{2}(x) \leq s_{n}^{2}:=n \sum_{k=0}^{n-1} \int_{0}^{\alpha_{1, \mathbf{Y}}(k)} Q^{2}(u) d u .
$$

Proof of Proposition 5.1 In all the proof, we shall use the following notation: for any nonnegative random variable $V$, let $Q_{V}$ be the generalized inverse of the tail function $x \rightarrow \mathbb{P}(V>x)$. We shall first prove Proposition 5.1 for $X_{i}=\sum_{\ell=1}^{L} a_{\ell} f_{\ell}\left(Y_{i}\right)-\sum_{\ell=1}^{L} a_{\ell} \mathbb{E}\left(f_{\ell}\left(Y_{i}\right)\right)$, with $f_{\ell}$ belonging to $\widetilde{\operatorname{Mon}}\left(Q, P_{Y_{0}}\right)$ and $\sum_{\ell=1}^{L}\left|a_{\ell}\right| \leq 1$. Let

$$
v=L_{n}(x) \text { and } M=Q(v) .
$$

Since $\alpha_{2, \mathbf{Y}}(0)=1 / 2$, it follows that $v \in[0,1 / 2]$. Note first that if $v=1 / 2$, then by using Markov's inequality, we derive

$$
\begin{aligned}
\mathbb{P}\left(\max _{1 \leq k \leq n}\left|S_{k}\right| \geq x\right) \leq \frac{1}{x} \sum_{k=1}^{n} \mathbb{E}\left(\left|X_{k}\right|\right) & \leq \frac{2}{x} \sum_{\ell=1}^{L}\left|a_{\ell}\right| \sum_{k=1}^{n} \mathbb{E}\left(\left|f_{\ell}\left(Y_{k}\right)\right|\right)=\frac{2 n}{x} \sum_{\ell=1}^{L}\left|a_{\ell}\right| \int_{0}^{1} Q_{\left|f_{\ell}\left(Y_{k}\right)\right|}(u) d u \\
\leq & \frac{2 n}{x} \int_{0}^{1} Q(u) d u \leq \frac{4 n}{x} \int_{0}^{1 / 2} Q(u) d u=\frac{4 n}{x} \int_{0}^{L_{n}(x)} Q(u) d u
\end{aligned}
$$

which then proves the proposition in case where $v=1 / 2$.

Therefore, we can assume in the rest of the proof that $v<1 / 2$. We then set $g_{M}(y)=(y \wedge M) \vee$ $(-M)$ where $M$ is defined in (5.3), and, for any $i \in \mathbb{Z}$ and any $\ell \geq 1$, we define

$$
Y_{i, \ell}^{\prime}=g_{M} \circ f_{\ell}\left(Y_{i}\right)-\mathbb{E}\left(g_{M} \circ f_{\ell}\left(Y_{i}\right)\right) \quad \text { and } \quad Y_{i, \ell}^{\prime \prime}=f_{\ell}\left(Y_{i}\right)-\mathbb{E}\left(f_{\ell}\left(Y_{i}\right)\right)-Y_{i, \ell}^{\prime} .
$$

Then, for any $i \in \mathbb{Z}$, we set

$$
X_{i}^{\prime}=\sum_{\ell=1}^{L} a_{\ell} Y_{i, \ell}^{\prime} \quad \text { and } \quad X_{i}^{\prime \prime}=X_{i}-X_{i}^{\prime} .
$$


Let $q=\min \left\{k \in \mathbb{N}: \alpha_{2, \mathbf{Y}}(k) \leq v\right\} \wedge n$ where $v$ in defined in (5.3). Since $R_{n}$ is right-continuous and non-increasing, we have $R_{n}\left(L_{n}(w)\right) \leq w$ for any $w$, hence

$$
q M=R_{n}(v)=R_{n}\left(L_{n}(x)\right) \leq x .
$$

Assume first that $q=n$. Bounding $X_{i}^{\prime}$ by $2 M$, we obtain $\max _{1 \leq k \leq n}\left|S_{k}\right| \leq 2 q M+\sum_{k=1}^{n}\left|X_{k}^{\prime \prime}\right|$. Taking into account (5.4) this gives

$$
\mathbb{P}\left(\max _{1 \leq k \leq n}\left|S_{k}\right| \geq 8 x\right) \leq \frac{1}{6 x} \sum_{k=1}^{n} \mathbb{E}\left(\left|X_{k}^{\prime \prime}\right|\right) .
$$

Writing $\varphi_{M}(x)=(|x|-M)_{+}$, we have

$$
\sum_{k=1}^{n} \mathbb{E}\left(\left|X_{k}^{\prime \prime}\right|\right) \leq 2 \sum_{\ell=1}^{L}\left|a_{\ell}\right| \sum_{k=1}^{n} \mathbb{E}\left(\varphi_{M}\left(f_{\ell}\left(Y_{k}\right)\right)\right)
$$

But $Q_{\varphi_{M}\left(f_{\ell}\left(Y_{k}\right)\right)} \leq Q_{\left|f_{\ell}\left(Y_{k}\right)\right|} \mathbf{1}_{[0, v]} \leq Q \mathbf{1}_{[0, v]}$. Consequently

$$
\sum_{k=1}^{n} \mathbb{E}\left(\left|X_{k}^{\prime \prime}\right|\right) \leq 2 \sum_{\ell=1}^{L}\left|a_{\ell}\right| \sum_{k=1}^{n} \int_{0}^{v} Q_{\left|f_{\ell}\left(Y_{k}\right)\right|}(u) d u \leq 2 n \int_{0}^{v} Q(u) d u \leq 2 n \int_{0}^{L_{n}(x)} Q(u) d u,
$$

where for the last inequality we have used that $u<v \Longleftrightarrow Q(v)<Q(u)$. We derive that

$$
\mathbb{P}\left(\max _{1 \leq k \leq n}\left|S_{k}\right| \geq 8 x\right) \leq \frac{n}{x} \int_{0}^{L_{n}(x)} Q(u) d u,
$$

which proves the result in case where $q=n$.

From now on, we assume that $q<n$. Therefore $q=\min \left\{k \in \mathbb{N}: \alpha_{2, \mathbf{Y}}(k) \leq v\right\}$ and then $\alpha_{2, \mathbf{Y}}(q) \leq v$. Recall also that since $v$ is assumed to be strictly less than $1 / 2$ then $q \geq 1$. For any integer $i$, define the random variables

$$
U_{i}=\sum_{k=(i-1) q+1}^{i q} X_{k}^{\prime}
$$

Consider now the $\sigma$-algebras $\mathcal{G}_{i}=\mathcal{F}_{i q}$ and define the variables $\tilde{U}_{i}$ as follows:

$$
\tilde{U}_{2 i-1}=U_{2 i-1}-\mathbb{E}\left(U_{2 i-1} \mid \mathcal{G}_{2(i-1)-1}\right) \quad \text { and } \quad \tilde{U}_{2 i}=U_{2 i}-\mathbb{E}\left(U_{2 i} \mid \mathcal{G}_{2(i-1)}\right) .
$$

The following inequality is then valid

$$
\max _{1 \leq k \leq n}\left|S_{k}\right| \leq 2 q M+\max _{2 \leq 2 j \leq[n / q]}\left|\sum_{i=1}^{j} \tilde{U}_{2 i}\right|+\max _{1 \leq 2 j-1 \leq[n / q]}\left|\sum_{i=1}^{j} \tilde{U}_{2 i-1}\right|+\sum_{i=1}^{[n / q]}\left|U_{i}-\tilde{U}_{i}\right|+\sum_{k=1}^{n}\left|X_{k}^{\prime \prime}\right| .
$$

(See the proof of Inequality (2.2) in [2]). Using (5.5) and (5.4), it follows that

$$
\begin{array}{r}
\mathbb{P}\left(\max _{1 \leq k \leq n}\left|S_{k}\right| \geq 8 x\right) \leq \mathbb{P}\left(\max _{2 \leq 2 j \leq[n / q]}\left|\sum_{i=1}^{j} \tilde{U}_{2 i}\right| \geq x\right)+\mathbb{P}\left(\max _{1 \leq 2 j-1 \leq[n / q]}\left|\sum_{i=1}^{j} \tilde{U}_{2 i-1}\right| \geq x\right) \\
+\mathbb{P}\left(\sum_{i=1}^{[n / q]}\left|U_{i}-\tilde{U}_{i}\right| \geq x\right)+\frac{n}{x} \int_{0}^{L_{n}(x)} Q(u) d u .
\end{array}
$$


Using Markov's inequality and stationarity, we get

$$
\mathbb{P}\left(\sum_{i=1}^{[n / q]}\left|U_{i}-\tilde{U}_{i}\right| \geq x\right) \leq \frac{1}{x} \sum_{i=1}^{[n / q]}\left\|\mathbb{E}\left(U_{i} \mid \mathcal{F}_{(i-2) q}\right)\right\|_{1}=\frac{n}{q x} \sum_{i=q+1}^{2 q}\left\|\mathbb{E}\left(X_{i}^{\prime} \mid \mathcal{F}_{0}\right)\right\|_{1} .
$$

Now, setting $A=\operatorname{sign}\left\{\mathbb{E}\left(X_{i}^{\prime} \mid \mathcal{F}_{0}\right)\right\}$,

$$
\left\|\mathbb{E}\left(X_{i}^{\prime} \mid \mathcal{F}_{0}\right)\right\|_{1}=\mathbb{E}\left((A-\mathbb{E} A) X_{i}^{\prime}\right)=\sum_{\ell=1}^{L} a_{\ell} \mathbb{E}\left((A-\mathbb{E} A)\left(g_{M} \circ f_{\ell}\left(Y_{i}\right)-\mathbb{E} g_{M} \circ f_{\ell}\left(Y_{i}\right)\right)\right) .
$$

Applying Theorem 1.1 in [16], and using the fact that $Q_{\left|g_{M} \circ f_{\ell}\left(Y_{i}\right)\right|}(u) \leq Q(u)$, we derive that

$$
\left|\mathbb{E}\left((A-\mathbb{E} A)\left(g_{M} \circ f_{\ell}\left(Y_{i}\right)-\mathbb{E} g_{M} \circ f_{\ell}\left(Y_{i}\right)\right)\right)\right| \leq 2 \int_{0}^{2 \bar{\alpha}\left(A, g_{M} \circ f_{\ell}\left(Y_{i}\right)\right)} Q(u) d u
$$

where, for any real-valued random variables $A, B$,

$$
\bar{\alpha}(A, B)=\sup _{(s, t) \in \mathbb{R}^{2}}\left|\operatorname{Cov}\left(\mathbf{1}_{A \leq s}, \mathbf{1}_{B \leq t}\right)\right| .
$$

Since for all $i \geq q$,

$$
\bar{\alpha}\left(A, g_{M} \circ f_{\ell}\left(Y_{i}\right)\right) \leq 2 \bar{\alpha}\left(A, Y_{i}\right) \leq \alpha_{1, \mathbf{Y}}(i) \leq \alpha_{2, \mathbf{Y}}(i)
$$

we derive

$$
\left\|\mathbb{E}\left(X_{i}^{\prime} \mid \mathcal{F}_{0}\right)\right\|_{1} \leq 4 \int_{0}^{\alpha_{2, \mathbf{Y}}(i)} Q(u) d u
$$

which implies

$$
\mathbb{P}\left(\sum_{i=1}^{[n / q]}\left|U_{i}^{\prime}-\tilde{U}_{i}\right| \geq x\right) \leq \frac{4 n}{x} \int_{0}^{\alpha_{2, \mathbf{Y}}(q)} Q(u) d u \leq \frac{4 n}{x} \int_{0}^{L_{n}(x)} Q(u) d u .
$$

Next, by using Markov's inequality,

$$
\mathbb{P}\left(\max _{2 \leq 2 j \leq[n / q]}\left|\sum_{i=1}^{j} \tilde{U}_{2 i}\right| \geq x\right) \leq\left.\frac{1}{x^{r}}\left\|\max _{2 \leq 2 j \leq[n / q]}\left|\sum_{i=1}^{j} \tilde{U}_{2 i}\right|\right\|\right|_{r} ^{r} .
$$

Note that $\left(\tilde{U}_{2 i}\right)_{i \in \mathbb{Z}}$ (resp. $\left.\left(\tilde{U}_{2 i-1}\right)_{i \in \mathbb{Z}}\right)$ is a stationary sequence of martingale differences with respect to the filtration $\left(\mathcal{G}_{2 i}\right)_{i \in \mathbb{Z}}$ (resp. $\left.\left(\mathcal{G}_{2 i-1}\right)_{i \in \mathbb{Z}}\right)$. By using the Rosenthal inequality given in [14] (see their Theorem 6), we get

$$
\left\|\max _{2 \leq 2 j \leq[n / q]}\left|\sum_{i=1}^{j} \tilde{U}_{2 i}\right|\right\|_{r} \ll(n / q)^{1 / r}\left\|\tilde{U}_{2}\right\|_{r}+(n / q)^{1 / r}\left(\sum_{k=1}^{[n / q]} \frac{1}{k^{1+2 \delta / r}}\left\|\mathbb{E}_{0}\left(\left(\sum_{i=1}^{k} \tilde{U}_{2 i}\right)^{2}\right)\right\|_{r / 2}^{\delta}\right)^{\frac{1}{2 \delta}}
$$

where $\delta=\min (1,1 /(r-2))$. Since $\left(\tilde{U}_{2 i}\right)_{i \in \mathbb{Z}}$ is a stationary sequence of martingale differences with respect to the filtration $\left(\mathcal{G}_{2 i}\right)_{i \in \mathbb{Z}}$,

$$
\mathbb{E}_{0}\left(\left(\sum_{i=1}^{k} \tilde{U}_{2 i}\right)^{2}\right)=\sum_{i=1}^{k} \mathbb{E}_{0}\left(\tilde{U}_{2 i}^{2}\right) .
$$


Moreover,

$$
\mathbb{E}_{0}\left(\tilde{U}_{2 i}^{2}\right) \leq \mathbb{E}_{0}\left(U_{2 i}^{2}\right)
$$

Therefore

$$
\left\|\mathbb{E}_{0}\left(\left(\sum_{i=1}^{k} \tilde{U}_{2 i}\right)^{2}\right)\right\|_{r / 2} \leq \sum_{i=1}^{k}\left\|\mathbb{E}_{0}\left(U_{2 i}^{2}\right)-\mathbb{E}\left(U_{2 i}^{2}\right)\right\|_{r / 2}+\sum_{i=1}^{k} \mathbb{E}\left(U_{2 i}^{2}\right)
$$

By stationarity

$$
\sum_{i=1}^{k} \mathbb{E}\left(U_{2 i}^{2}\right)=k\left\|S_{q}^{\prime}\right\|_{2}^{2}, \text { with } S_{q}^{\prime}=\sum_{i=1}^{q} X_{i}^{\prime}
$$

Moreover

$$
\left\|\tilde{U}_{2}\right\|_{r} \leq 2\left\|S_{q}^{\prime}\right\|_{r}
$$

It follows that

$$
\left\|\max _{2 \leq 2 j \leq[n / q]}\left|\sum_{i=1}^{j} \tilde{U}_{2 i}(t)\right|\right\|_{r}^{r} \ll \frac{n}{q}\left\|S_{q}^{\prime}\right\|_{r}^{r}+\left(\frac{n}{q}\right)^{r / 2}\left\|S_{q}^{\prime}\right\|_{2}^{r}+\frac{n}{q}\left(\sum_{k=1}^{[n / q]} \frac{1}{k^{1+2 \delta / r}} D_{k, q}^{\delta}\right)^{r /(2 \delta)},
$$

where

$$
D_{k, q}=\sum_{i=1}^{k}\left\|\mathbb{E}_{0}\left(U_{2 i}^{2}\right)-\mathbb{E}\left(U_{2 i}^{2}\right)\right\|_{r / 2}
$$

Notice that

$$
\begin{aligned}
\left\|\mathbb{E}_{0}\left(U_{2 i}^{2}\right)-\mathbb{E}\left(U_{2 i}^{2}\right)\right\|_{r / 2} \leq & \sum_{j, k=(2 i-1) q+1}^{2 i q}\left\|\mathbb{E}_{0}\left(X_{j}^{\prime} X_{k}^{\prime}\right)-\mathbb{E}\left(X_{j}^{\prime} X_{k}^{\prime}\right)\right\|_{r / 2} \\
& \leq \sum_{\ell, m=1}^{L} \sum_{j, k=(2 i-1) q+1}^{2 i q}\left|a_{\ell}\left\|a_{m} \mid\right\| \mathbb{E}_{0}\left(Y_{j, \ell}^{\prime} Y_{k, m}^{\prime}\right)-\mathbb{E}\left(Y_{j, \ell}^{\prime} Y_{k, m}^{\prime}\right) \|_{r / 2} .\right.
\end{aligned}
$$

Now setting

$$
Z:=\left|\mathbb{E}_{0}\left(Y_{j, \ell}^{\prime} Y_{k, m}^{\prime}\right)-\mathbb{E}\left(Y_{j, \ell}^{\prime} Y_{k, m}^{\prime}\right)\right|^{r / 2-1} \operatorname{sign}\left\{\mathbb{E}_{0}\left(Y_{j, \ell}^{\prime} Y_{k, m}^{\prime}\right)-\mathbb{E}\left(Y_{j, \ell}^{\prime} Y_{k, m}^{\prime}\right)\right\}
$$

we get

$$
\begin{array}{r}
\left\|\mathbb{E}_{0}\left(Y_{j, \ell}^{\prime} Y_{k, m}^{\prime}\right)-\mathbb{E}\left(Y_{j, \ell}^{\prime} Y_{k, m}^{\prime}\right)\right\|_{r / 2}^{r / 2}=\mathbb{E}\left(Z\left(\mathbb{E}_{0}\left(Y_{j, \ell}^{\prime} Y_{k, m}^{\prime}\right)-\mathbb{E}\left(Y_{j, \ell}^{\prime} Y_{k, m}^{\prime}\right)\right)\right) \\
=\mathbb{E}\left((Z-\mathbb{E}(Z)) Y_{j, \ell}^{\prime} Y_{k, m}^{\prime}\right) .
\end{array}
$$

From Proposition A.1 and Lemma A.1 in [7], noticing that $Q_{\left|g_{M} \circ f_{\ell}\left(Y_{i}\right)\right|}(u) \leq M$, we derive

$$
\mathbb{E}\left((Z-\mathbb{E}(Z)) Y_{j, \ell}^{\prime} Y_{k, m}^{\prime}\right) \leq 2^{4} M^{2} \int_{0}^{\bar{\alpha} / 2} Q_{|Z|}(u) d u
$$

with $\bar{\alpha}:=\alpha\left(Z, g_{M} \circ f_{\ell}\left(Y_{j}\right), g_{M} \circ f_{m}\left(Y_{k}\right)\right)$, where for real-valued random variables $Z, B, V$,

$$
\bar{\alpha}(Z, B, V)=\sup _{(s, t, u) \in \mathbf{R}^{3}}\left|\mathbb{E}\left(\left(\mathbf{1}_{Z \leq s}-\mathbb{P}(Z \leq s)\right)\left(\mathbf{1}_{B \leq t}-\mathbb{P}(B \leq t)\right)\left(\mathbf{1}_{V \leq u}-\mathbb{P}(V \leq u)\right)\right)\right| .
$$


Applying Hölder's inequality,

$$
\int_{0}^{\bar{\alpha} / 2} Q_{|Z|}(u) d u \leq(\bar{\alpha} / 2)^{2 / r}\left(\int_{0}^{1} Q_{|Z|}^{r /(r-2)}(u)\right)^{(r-2) / r}=(\bar{\alpha} / 2)^{2 / r}\|Z\|_{r /(r-2)} .
$$

Since

$$
\|Z\|_{r /(r-2)}=\left\|\mathbb{E}_{0}\left(Y_{j, \ell}^{\prime} Y_{k, m}^{\prime}\right)-\mathbb{E}\left(Y_{j, \ell}^{\prime} Y_{k, m}^{\prime}\right)\right\|_{r / 2}^{r / 2-1}
$$

we then derive

$$
\left\|\mathbb{E}_{0}\left(Y_{j, \ell}^{\prime} Y_{k, m}^{\prime}\right)-\mathbb{E}\left(Y_{j, \ell}^{\prime} Y_{k, m}^{\prime}\right)\right\|_{r / 2} \leq 2^{4} M^{2}(\bar{\alpha} / 2)^{2 / r} .
$$

Now, for all $j, k \geq(2 i-1) q+1$,

$$
\bar{\alpha} \leq 4 \bar{\alpha}\left(Z, f_{\ell}\left(Y_{j}\right), f_{\ell}\left(Y_{k}\right)\right) \leq 2 \alpha_{2, \mathbf{Y}}((2 i-q)+1) .
$$

So, overall, using the fact that $\sum_{\ell=1}^{L}\left|a_{\ell}\right| \leq 1$ and that $\alpha_{2, \mathbf{Y}}((2 i-q)+1) \leq \alpha_{2, \mathbf{Y}}(i q+1)$, we get

$$
D_{k, q} \leq 2^{4} q^{2} M^{2} \sum_{i=1}^{k} \alpha_{2, \mathbf{Y}}^{2 / r}(i q+1) .
$$

Let $\eta=(\beta-2) / r$ and recall that $r-2<\beta<r$. Since $\eta<(r-2) / r$, applying Hölder's inequality, we then get

$$
D_{k, q} \ll q^{2} M^{2} k^{-\eta+(r-2) / r}\left(\sum_{i=1}^{k} i^{\beta / 2-1} \alpha_{2, \mathbf{Y}}(i q+1)\right)^{2 / r} .
$$

Since $2 \delta / r>-\delta \eta+\delta(r-2) / r$ (indeed $-\eta+(r-2) / r=(r-\beta) / r$ and $r-\beta<2$ ), it follows that

$$
\sum_{k=1}^{[n / q]} \frac{1}{k^{1+2 \delta / r}} D_{k, q}^{\delta} \ll(q M)^{2 \delta}\left(\sum_{i=1}^{[n / q]} i^{\beta / 2-1} \alpha_{2, \mathbf{Y}}(i q+1)\right)^{2 \delta / r}
$$

But, since $x<\alpha_{2, \mathbf{Y}}^{-1}(u) \Longleftrightarrow \alpha_{2, \mathbf{Y}}(x)>u$ and $\alpha_{2, \mathbf{Y}}(q) \leq v$ (since $q<n$ ),

$$
\begin{aligned}
\sum_{i=1}^{[n / q]} i^{\beta / 2-1} \alpha_{2, \mathbf{Y}}(i q+1)= & \sum_{i=1}^{[n / q]} i^{\beta / 2-1} \int_{0}^{1} \mathbf{1}_{u<\alpha_{2, \mathbf{Y}}(i q+1)} d u \\
& \leq \int_{0}^{v} \sum_{i=1}^{[n / q]} i^{\beta / 2-1} \mathbf{1}_{i \leq q^{-1} \alpha_{2, \mathbf{Y}}^{-1}(u)} d u \leq q^{-\beta / 2} \int_{0}^{v}\left(\alpha_{2, \mathbf{Y}}^{-1}(u) \wedge n\right)^{\beta / 2} d u .
\end{aligned}
$$

So, overall,

$$
\sum_{k=1}^{[n / q]} \frac{1}{k^{1+2 \delta / r}} D_{k, q}^{\delta} \ll(q M)^{2 \delta} q^{-\beta \delta / r}\left(\int_{0}^{v}\left(\alpha_{2, \mathbf{Y}}^{-1}(u) \wedge n\right)^{\beta / 2} d u\right)^{2 \delta / r},
$$

which implies that

$$
\frac{n}{q x^{r}}\left(\sum_{k=1}^{[n / q]} \frac{1}{k^{1+2 \delta / r}} D_{k, q}^{\delta}\right)^{r /(2 \delta)} \ll \frac{n}{x^{r}}(q M)^{r} q^{-\beta / 2-1} \int_{0}^{v}\left(\alpha_{2, \mathbf{Y}}^{-1}(u) \wedge n\right)^{\beta / 2} d u .
$$


Using the fact that $r-\beta / 2-1>0$ (since $\beta<r$ and $r>2$ ) and (5.4), we infer that $(q M)^{r} q^{-\beta / 2-1} \leq x^{r-\beta / 2-1} Q^{\beta / 2+1}(v)$. Moreover, $u<v \Longleftrightarrow Q(v)<Q(u)$. Therefore

$$
\frac{n}{q x^{r}}\left(\sum_{k=1}^{[n / q]} \frac{1}{k^{1+2 \delta / r}} D_{k, q}^{\delta}\right)^{r /(2 \delta)} \ll \frac{n}{x^{\beta / 2+1}} \int_{0}^{v}\left(\alpha_{2, \mathbf{Y}}^{-1}(u) \wedge n\right)^{\beta / 2} Q^{\beta / 2+1}(u) d u .
$$

We prove now that

$$
\frac{n}{q x^{r}}\left\|S_{q}^{\prime}\right\|_{r}^{r} \ll \frac{n}{x} \int_{0}^{L_{n}(x)} Q(u) d u+\frac{n}{x^{1+r / 2}} \int_{L_{n}(x)}^{1} R_{n}^{r / 2}(u) Q(u) d u .
$$

Using the stationarity and applying Theorem 2.5(a) in [16], we get

$$
\left\|S_{q}^{\prime}\right\|_{r} \leq \sum_{\ell=1}^{L}\left|a_{\ell}\right|\left\|\sum_{i=1}^{q} Y_{i, \ell}^{\prime}\right\|_{r} \leq \sum_{\ell=1}^{L}\left|a_{\ell}\right|\left(2 q r \max _{1 \leq k \leq q}\left\|Y_{0, \ell}^{\prime} \sum_{i=0}^{k-1} \mathbb{E}_{0}\left(Y_{i, \ell}^{\prime}\right)\right\|_{r / 2}\right)^{1 / 2} .
$$

Setting

$$
W_{0, k}:=\left|Y_{0, \ell}^{\prime} \sum_{i=0}^{k-1} \mathbb{E}_{0}\left(Y_{i, \ell}^{\prime}\right)\right|^{r / 2-1} \operatorname{sign}\left\{Y_{0, \ell}^{\prime} \sum_{j=0}^{k-1} \mathbb{E}_{0}\left(Y_{j, \ell}^{\prime}\right)\right\}
$$

we get

$$
\left\|Y_{0, \ell}^{\prime} \sum_{i=0}^{k-1} \mathbb{E}_{0}\left(Y_{i, \ell}^{\prime}\right)\right\|_{r / 2}^{r / 2}=\sum_{j=0}^{k-1} \mathbb{E}\left(W_{0, k} Y_{0, \ell}^{\prime} \mathbb{E}_{0}\left(Y_{j, \ell}^{\prime}\right)\right)=\sum_{j=0}^{k-1} \mathbb{E}\left(\left(W_{0, k} Y_{0, \ell}^{\prime}-\mathbb{E}\left(W_{0, k} Y_{0, \ell}^{\prime}\right)\right) g_{M} \circ f_{\ell}\left(Y_{j}\right)\right) .
$$

From Proposition A.1 and Lemma A.1 in [7], noticing that $Q_{\left|g_{M} \circ f_{\ell}\left(Y_{j}\right)\right|}(u) \leq Q(u \vee v)$, we derive

$$
\begin{aligned}
\mathbb{E}\left(\left(W_{0, k} Y_{0, \ell}^{\prime}-\mathbb{E}\left(W_{0, k} Y_{0, \ell}^{\prime}\right)\right) g_{M} \circ f_{\ell}\left(Y_{j}\right)\right) \leq 4 \int_{0}^{\tilde{\alpha} / 2} Q_{\left|W_{0, k} Y_{0, \ell}^{\prime}\right|}(u) Q(u \vee v) d u \\
\leq 8 \int_{0}^{\tilde{\alpha} / 2} Q_{\left|W_{0, k}\right|}(u) Q^{2}(u \vee v) d u,
\end{aligned}
$$

where

$$
\tilde{\alpha}:=\sup _{(s, t) \in \mathbf{R}^{2}}\left|\mathbb{E}\left(\left(\mathbf{1}_{W_{0, k} Y_{0, \ell}^{\prime} \leq s}-\mathbb{P}\left(W_{0, k} Y_{0, \ell}^{\prime} \leq s\right)\right)\left(\mathbf{1}_{g_{M} \circ f_{\ell}\left(Y_{j}\right) \leq t}-\mathbb{P}\left(g_{M} \circ f_{\ell}\left(Y_{j}\right) \leq t\right)\right)\right)\right|
$$

Now, for any $j \geq 0$,

$\bar{\alpha} \leq 2 \sup _{(s, t) \in \mathbf{R}^{2}}\left|\mathbb{E}\left(\left(\mathbf{1}_{W_{0, k} Y_{0, \ell}^{\prime} \leq s}-\mathbb{P}\left(W_{0, k} Y_{0, \ell}^{\prime} \leq s\right)\right)\left(\mathbf{1}_{f_{\ell}\left(Y_{j}\right) \leq t}-\mathbb{P}\left(f_{\ell}\left(Y_{j}\right) \leq t\right)\right)\right)\right| \leq 2 \alpha_{1, \mathbf{Y}}(j) \leq 2 \alpha_{2, \mathbf{Y}}(j)$.

It follows that

$$
\left\|Y_{0, \ell}^{\prime} \sum_{i=0}^{k-1} \mathbb{E}_{0}\left(Y_{i, \ell}^{\prime}\right)\right\|_{r / 2}^{r / 2} \leq 8 \sum_{j=0}^{k-1} \int_{0}^{1} \mathbf{1}_{u<\alpha_{2, \mathbf{Y}}(j)} Q_{\left|W_{0, k}\right|}(u) Q^{2}(u \vee v) d u
$$


Notice that $\sum_{j=0}^{k-1} \mathbf{1}_{u<\alpha_{2, \mathbf{Y}}(j)}=\alpha_{2, \mathbf{Y}}^{-1}(u) \wedge k$. Applying Hölder's inequality, we then get

$$
\begin{aligned}
\sum_{j=0}^{k-1} \int_{0}^{1} \mathbf{1}_{u<\alpha_{2, \mathbf{Y}}(j)} Q_{\left|W_{0, k}\right|}(u) & Q^{2}(u \vee v) d u \\
\leq\left(\int _ { 0 } ^ { 1 } \left(\alpha_{2, \mathbf{Y}}^{-1}(u)\right.\right. & \left.\wedge k)^{r / 2} Q^{r}(u \vee v) d u\right)^{2 / r}\left(\int_{0}^{1} Q_{\left|W_{0, k}\right|}^{r /(r-2)}(u) d u\right)^{(r-2) / r} \\
\leq & \left(\int_{0}^{1}\left(\alpha_{2, \mathbf{Y}}^{-1}(u) \wedge k\right)^{r / 2} Q^{r}(u \vee v) d u\right)^{2 / r}\left\|Y_{0, \ell}^{\prime} \sum_{i=0}^{k-1} \mathbb{E}_{0}\left(Y_{i, \ell}^{\prime}\right)\right\|_{r / 2}^{r / 2-1} .
\end{aligned}
$$

So, overall,

$$
\left\|Y_{0, \ell}^{\prime} \sum_{i=0}^{k-1} \mathbb{E}_{0}\left(Y_{i, \ell}^{\prime}\right)\right\|_{r / 2} \leq 8\left(\int_{0}^{1}\left(\alpha_{2, \mathbf{Y}}^{-1}(u) \wedge k\right)^{r / 2} Q^{r}(u \vee v) d u\right)^{2 / r}
$$

Taking into account this bound in (5.13) and using the fact that $\sum_{\ell=1}^{L}\left|a_{L}\right| \leq 1$, we derive

$$
\left\|S_{q}^{\prime}\right\|_{r}^{r} \leq(16 r q)^{r / 2} \int_{0}^{1}\left(\alpha_{2, \mathbf{Y}}^{-1}(u) \wedge q\right)^{r / 2} Q^{r}(u \vee v) d u
$$

Since $\alpha_{2, \mathbf{Y}}^{-1}(u) \wedge q=q \mathbf{1}_{0<u \leq v}+\left(\alpha_{2, \mathbf{Y}}^{-1}(u) \wedge n\right) \mathbf{1}_{v<u \leq 1}$, the above upper bound gives

$$
\frac{n}{q x^{r}}\left\|S_{q}^{\prime}\right\|_{r}^{r} \ll \frac{n}{x^{r}}(q Q(v))^{r-1} \int_{0}^{v} Q(u) d u+\frac{n}{x^{r}}(q M)^{r / 2-1} \int_{v}^{1}\left(\alpha_{2, \mathbf{Y}}^{-1}(u) \wedge n\right)^{r / 2} Q^{r / 2+1}(u) d u,
$$

which proves (5.12) by taking into account (5.4).

We show now that

$$
\frac{n^{r / 2}}{q^{r / 2} x^{r}}\left\|S_{q}^{\prime}\right\|_{2}^{r} \ll x^{-r} s_{n}^{r}(x)+\left(\frac{n}{x} \int_{0}^{L_{n}(x)} Q(u) d u\right)^{r / 2} .
$$

By stationarity

$$
\mathbb{E}\left(S_{q}^{\prime}\right)^{2}=\sum_{|i| \leq q}(q-|i|) \mathbb{E}\left(X_{0}^{\prime} X_{|i|}^{\prime}\right) .
$$

Now, by Inequality (2.5) in [2],

$$
\mathbb{E}\left(X_{0}^{\prime} X_{|i|}^{\prime}\right) \leq 4 \int_{0}^{\alpha_{1, \mathbf{Y}}(|i|)} Q^{2}(u \vee v) d u
$$

so that

$$
\mathbb{E}\left(S_{q}^{\prime}\right)^{2} \leq 8 q \sum_{i=0}^{q-1} \int_{0}^{\alpha_{1, \mathbf{Y}}(i)} Q^{2}(u \vee v) d u=8 q \int_{0}^{1}\left(\alpha_{1, \mathbf{Y}}^{-1}(u) \wedge q\right) Q^{2}(u \vee v) d u
$$

Recall that $v=L_{n}(x)$ and that $q Q(v) \leq x$, by (5.4). Using in addition the fact that $\alpha_{1, \mathbf{Y}}^{-1} \leq \alpha_{2, \mathbf{Y}}^{-1}$, we get

$$
\mathbb{E}\left(S_{q}^{\prime}\right)^{2} \leq 8 q \int_{L_{n}(x)}^{1}\left(\alpha_{1, \mathbf{Y}}^{-1}(u) \wedge n\right) Q^{2}(u) d u+8 q x \int_{0}^{L_{n}(x)} Q(u) d u,
$$


which proves (5.14).

Starting from (5.9) and taking into account (5.10), (5.11), (5.12) and (5.14), we get

$$
\begin{aligned}
\mathbb{P}\left(\max _{2 \leq 2 j \leq[n / q]}\left|\sum_{i=1}^{j} \tilde{U}_{2 i}\right| \geq x\right) \ll \frac{s_{n}^{r}}{x^{r}}+\frac{n}{x} \int_{0}^{L_{n}(x)} Q(u) d u & +\frac{n}{x^{1+\beta / 2}} \int_{0}^{L_{n}(x)} R_{n}^{\beta / 2}(u) Q(u) d u \\
& +\frac{n}{x^{1+r / 2}} \int_{L_{n}(x)}^{1} R_{n}^{r / 2}(u) Q(u) d u
\end{aligned}
$$

Note that, to deal with the second term on right hand in (5.14), we have used the fact if

$$
\frac{n}{x} \int_{0}^{L_{n}(x)} Q(u) d u \geq 1
$$

then the bound (5.15) is trivial, and otherwise

$$
\left(\frac{n}{x} \int_{0}^{L_{n}(x)} Q(u) d u\right)^{r / 2} \leq \frac{n}{x} \int_{0}^{L_{n}(x)} Q(u) d u .
$$

Obviously the upper bound (5.15) is also valid for the quantity

$$
\mathbb{P}\left(\max _{1 \leq 2 j+1 \leq[n / q]}\left|\sum_{i=1}^{j} \tilde{U}_{2 i+1}\right| \geq x\right) .
$$

Together with (5.6) and (5.8) this completes the proof of the proposition for $q<n$.

The proposition is proved for any variable $X_{i}=f\left(Y_{i}\right)-\mathbb{E}\left(f\left(Y_{i}\right)\right)$ with $f=\sum_{\ell=1}^{L} a_{\ell} f_{\ell}$ and $f_{\ell} \in \widetilde{\operatorname{Mon}}\left(Q, P_{Y_{0}}\right), \sum\left|a_{\ell}\right| \leq 1$. Let us explain how it can be extended to $\widetilde{\mathcal{F}}\left(Q, P_{Y_{0}}\right)$.

Let $f \in \widetilde{\mathcal{F}}\left(Q, P_{Y_{0}}\right)$. By definition of $\widetilde{\mathcal{F}}\left(Q, P_{Y_{0}}\right)$, there exists $f_{L}=\sum_{\ell=1}^{L} a_{\ell, L} g_{\ell, L}$ with $g_{\ell, L}$ belonging to $\widetilde{\operatorname{Mon}}\left(Q, P_{Y_{0}}\right)$ and $\sum_{\ell=1}^{L}\left|a_{\ell, L}\right| \leq 1$, and such that $f_{L}$ converges in $\mathbb{L}^{1}\left(P_{Y_{0}}\right)$ to $f$. It follows that $X_{i, L}=f_{L}\left(Y_{i}\right)-\mathbb{E}\left(f_{L}\left(Y_{i}\right)\right)$ converges in $\mathbb{L}^{1}$ to $X_{i}$ as $L$ tends to infinity. Extracting a subsequence if necessary, one may also assume that the convergence holds almost surely. Hence, for any fixed $n, S_{n, L}=\sum_{k=1}^{n} X_{k, L}$ converges almost surely and in $\mathbb{L}^{1}$ to $S_{n}$.

Let $Z_{n}=\max _{1 \leq k \leq n}\left|S_{k}\right|$. By Beppo-Levi,

$$
\mathbb{P}\left(\max _{1 \leq k \leq n}\left|S_{k}\right|>x\right)=\lim _{k \rightarrow \infty} \mathbb{P}\left(Z_{n}>x+k^{-1}\right) .
$$

Let $h_{k}$ be a continuous function from $\mathbb{R}$ to $[0,1]$, such that $h_{k}(t)=1$ if $t>x+k^{-1}$ and $h_{k}(t)=0$ if $t<x$. Let $Z_{n, L}=\max _{1 \leq k \leq n}\left|S_{k, L}\right|$. By Fatou's lemma,

$$
\mathbb{P}\left(Z_{n}>x+k^{-1}\right) \leq \mathbb{E}\left(h_{k}\left(Z_{n}\right)\right) \leq \liminf _{L \rightarrow \infty} \mathbb{E}\left(h_{k}\left(Z_{n, L}\right)\right) \leq \liminf _{L \rightarrow \infty} \mathbb{P}\left(Z_{n, L}>x\right) .
$$

Now, we have just proved that $\mathbb{P}\left(Z_{n, L}>x\right)$ satisfies the upper bound (5.1), uniformly in $L$. From (5.16) and (5.17), we infer that $\mathbb{P}\left(\max _{1 \leq k \leq n}\left|S_{k}\right|>x\right)$ satisfies also (5.1), which completes the proof of Proposition 5.1. 


\section{Proofs of the results of Section 3}

\subsection{Preparatory material}

Lemma 6.1. Let $p>1$. Assume that (3.5) holds and let $b \in(0, p-1)$ and $c>p-1$. Then $\sup _{x>0} x^{p-1-b} \int_{0}^{1} R^{b}(u) Q(u) \mathbf{1}_{R(u)>x} d u<\infty, \quad$ and $\sup _{x>0} \frac{1}{x^{c-p+1}} \int_{0}^{1} R^{c}(u) Q(u) \mathbf{1}_{R(u) \leq x} d u<\infty$.

Lemma 6.2. Let $p>1$. Assume that (3.16) holds and let $b \in(0, p-1)$ and $c>p-1$. Then $\lim _{x \rightarrow \infty} x^{p-1-b} \int_{0}^{1} R^{b}(u) Q(u) \mathbf{1}_{R(u)>x} d u=0, \quad$ and $\quad \lim _{x \rightarrow \infty} \frac{1}{x^{c-p+1}} \int_{0}^{1} R^{c}(u) Q(u) \mathbf{1}_{R(u) \leq x} d u=0$.

Proof. Let $Z$ be a random variable with values in $(0,1)$, whose distribution has density

$$
u \rightarrow \bar{Q}(u)=\frac{Q(u)}{\int_{0}^{1} Q(u) d u} .
$$

Then Condition (3.5) is equivalent to

$$
\sup _{x>0} x^{p-1} \mathbb{P}(R(Z)>x)<\infty
$$

and Condition (3.16) is equivalent to

$$
\lim _{x \rightarrow \infty} x^{p-1} \mathbb{P}(R(Z)>x)=0 .
$$

In the same way, the first statements of Lemmas 6.1 and 6.2 read respectively

$$
\sup _{x>0} x^{p-1-b} \mathbb{E}\left((R(Z))^{b} \mathbf{1}_{R(Z)>x}\right)<\infty, \quad \text { and } \quad \lim _{x \rightarrow \infty} x^{p-1-b} \mathbb{E}\left((R(Z))^{b} \mathbf{1}_{R(Z)>x}\right)=0 .
$$

Applying Fubini's theorem, one easily sees that

$$
\mathbb{E}\left((R(Z))^{b} \mathbf{1}_{R(Z)>x}\right)=x^{b} \mathbb{P}(R(Z)>x)+b \int_{x}^{\infty} u^{b-1} \mathbb{P}(R(Z)>u) d u .
$$

Now, (6.1) combined with (6.4) implies the first part of (6.3), and (6.2) combined with (6.4) implies the second part of (6.3). This concludes the proofs of the first statements of Lemmas 6.1 and 6.2 .

To prove the second statements of Lemmas 6.1 and 6.2, we first note that they are respectively equivalent to

$$
\sup _{x>0} \frac{1}{x^{c-p+1}} \mathbb{E}\left((R(Z))^{c} \mathbf{1}_{R(Z) \leq x}\right)<\infty, \quad \text { and } \quad \lim _{x \rightarrow \infty} \frac{1}{x^{c-p+1}} \mathbb{E}\left((R(Z))^{c} \mathbf{1}_{R(Z) \leq x}\right)=0 .
$$

Applying Fubini's theorem, one easily sees that

$$
\mathbb{E}\left((R(Z))^{c} \mathbf{1}_{R(Z) \leq x}\right)=c \int_{0}^{\infty} u^{c-1} \mathbb{P}(u<R(Z) \leq x) d u \leq c \int_{0}^{x} u^{c-1} \mathbb{P}(R(Z)>u) d u .
$$

Now, (6.1) combined with (6.6) implies the first part of (6.5), and (6.2) combined with (6.6) implies the second part of (6.5). This concludes the proofs of of Lemmas 6.1 and 6.2. 


\subsection{Proof of Theorem 3.1}

To prove (3.2), it suffices to write

$$
\left\|\max _{1 \leq k \leq n}\left|S_{k}\right|\right\|_{p}^{p}=p \int_{0}^{\infty} x^{p-1} \mathbb{P}\left(\max _{1 \leq k \leq n}\left|S_{k}\right| \geq x\right) d x \leq s_{n}^{p}+p \int_{s_{n}}^{\infty} x^{p-1} \mathbb{P}\left(\max _{1 \leq k \leq n}\left|S_{k}\right| \geq x\right) d x,
$$

and to apply Inequality (5.1) with $r-2<\beta<2 p-2<r<2 p$ to bound the last integral. The result follows by applying Fubini's theorem.

\subsection{Proof of Theorem 3.2}

We apply Proposition 5.1 with $r / 2=a \in(p-1, p)$ and $\beta \in(r-2,2 p-2)$.

Proof of Item 1. We start by proving (3.6). Recall that $s_{n}^{2}$ has been defined in (5.2) and that $s_{n}^{2}(x) \leq s_{n}^{2}$. We have that

$$
s_{n}^{2} \leq C(\alpha, Q) n, \quad \text { with } \quad C(\alpha, Q)=\sum_{i=0}^{\infty} \int_{0}^{\alpha_{1, \mathbf{Y}}(i)} Q^{2}(u) d u .
$$

Since $p>2$, the condition (3.5) implies that $C(\alpha, Q)<\infty$. Inequality (5.1) and Remark 5.2 yield

$$
\begin{aligned}
\mathbb{P}\left(\frac{1}{n} \max _{1 \leq k \leq n}\left|S_{k}\right| \geq x\right) \ll & \frac{1}{n^{a} x^{2 a}}+\frac{1}{x} \int_{0}^{1} Q(u) \mathbf{1}_{R(u)>n x} d u \\
& +\frac{1}{n^{\beta / 2} x^{1+\beta / 2}} \int_{0}^{1} R^{\beta / 2}(u) Q(u) \mathbf{1}_{R(u)>n x} d u \\
& \quad+\frac{1}{n^{r / 2} x^{1+r / 2}} \int_{0}^{1} R^{r / 2}(u) Q(u) \mathbf{1}_{R(u) \leq n x} d u .
\end{aligned}
$$

From (3.5), we infer that

$$
\frac{1}{x} \int_{0}^{1} Q(u) \mathbf{1}_{R(u)>n x} d u \ll \frac{1}{n^{p-1} x^{p}} .
$$

To handle the two last terms on right hand in (6.8), we apply Lemma 6.1. We then infer that

$$
\begin{aligned}
& \frac{1}{n^{\beta / 2} x^{1+\beta / 2}} \int_{0}^{1} R^{\beta / 2}(u) Q(u) \mathbf{1}_{R(u)>n x} d u \ll \frac{1}{n^{p-1} x^{p}}, \\
& \frac{1}{n^{r / 2} x^{1+r / 2}} \int_{0}^{1} R^{r / 2}(u) Q(u) \mathbf{1}_{R(u) \leq n x} d u \ll \frac{1}{n^{p-1} x^{p}},
\end{aligned}
$$

and (3.6) follows.

The proof of (3.7) is almost identical. The only difference is about the first term on right hand in (6.8). This first term is equal to $(n x)^{-2 a} s_{n}^{2 a}(n x)$, and for $c \in(0,1)$, one has

$$
\frac{s_{n}^{2 a}(n x)}{(n x)^{2 a}} \leq \frac{1}{n^{a} x^{2 a}}\left(\int Q(u) R^{c}(u) R^{1-c}(u) \mathbf{1}_{R(u) \leq n x} d u\right)^{a} .
$$

Since $p=2$, it follows that $\int Q(u) R^{c}(u) d u<\infty$, and finally

$$
\frac{s_{n}^{2 a}(n x)}{(n x)^{2 a}} \ll \frac{1}{n^{a c} x^{a(1+c)}},
$$


completing the proof of (3.7).

Proof of Item 2. We start from (6.8). Since the series with terms $n^{p-2}$ and $n^{p-2-(\beta / 2)}$ are divergent, we obtain by summing in $n$,

$$
\sum_{n=1}^{\infty} \frac{n^{p-2}}{x} \int_{0}^{1} Q(u) \mathbf{1}_{R(u)>n x} d u \ll \frac{1}{x^{p}} \int_{0}^{1}\left(\alpha_{2, \mathbf{Y}}^{-1}(u)\right)^{p-1} Q^{p}(u) d u .
$$

and

$$
\sum_{n=1}^{\infty} \frac{n^{p-2}}{n^{\beta / 2} x^{1+\beta / 2}} \int_{0}^{1} R^{\beta / 2}(u) Q(u) \mathbf{1}_{R(u)>n x} d u \ll \frac{1}{x^{p}} \int_{0}^{1}\left(\alpha_{2, \mathbf{Y}}^{-1}(u)\right)^{p-1} Q^{p}(u) d u .
$$

Since the series with term $n^{p-2-(r / 2)}$ converges, we obtain by summing in $n$,

$$
\sum_{n=1}^{\infty} \frac{n^{p-2}}{n^{r / 2} x^{1+r / 2}} \int_{0}^{1} R^{r / 2}(u) Q(u) \mathbf{1}_{R(u) \leq n x} d u \ll \frac{1}{x^{p}} \int_{0}^{1}\left(\alpha_{2, \mathbf{Y}}^{-1}(u)\right)^{p-1} Q^{p}(u) d u .
$$

The proof of Item 2 is now complete.

\subsection{Proof of Theorem 3.3}

Since $p>2$, the condition (3.16) implies (3.14), and the process $\left\{W_{n}(t), t \in[0,1]\right\}$ converges in distribution in the space $C\left([0,1],\|\cdot\|_{\infty}\right)$ to $\sigma W$. It remains to prove the tightness in $\mathcal{H}_{\delta}^{0}([0,1])$. We start from the tightness criterion given in [8]: the process $\left\{W_{n}(t), t \in[0,1]\right\}$ is tight in $\mathcal{H}_{\delta}^{0}([0,1])$ as soon as, for any $\varepsilon>0$,

$$
\lim _{\eta \rightarrow 0} \limsup _{n \rightarrow \infty} n \sum_{k=1}^{[\log (n \eta)]} \frac{1}{2^{k}} \mathbb{P}\left(\max _{1 \leq i \leq 2^{k}}\left|S_{i}\right|>\varepsilon 2^{k \delta} n^{1 / p}\right)=0,
$$

where $\log$ is the binary logarithm. Applying Proposition 5.1 with $s_{n}$ as in (6.7), $r \in(2 p-2,2 p)$, and $\beta \in(r-2,2 p-2)$, we get that

$$
n \sum_{k=1}^{[\log (n \eta)]} \frac{1}{2^{k}} \mathbb{P}\left(\max _{1 \leq i \leq 2^{k}}\left|S_{i}\right|>\varepsilon 2^{k \delta} n^{1 / p}\right) \leq \sum_{i=1}^{4} I_{i}(n, \eta),
$$

with

$$
\begin{aligned}
& I_{1}(n, \eta) \ll \frac{1}{n^{(r-p) / p}} \sum_{k=1}^{[\log (n \eta)]} 2^{k(r-p) / p}, \\
& I_{2}(n, \eta) \ll \sum_{k=1}^{\infty} \frac{n^{(p-1) / p}}{2^{k \delta}} \int_{0}^{1} Q(u) \mathbf{1}_{R(u)>\varepsilon 2^{k \delta} n^{1 / p}} d u, \\
& I_{3}(n, \eta) \ll \sum_{k=1}^{\infty} \frac{n^{(2 p-2-\beta) / 2 p}}{2^{k \delta(2+\beta) / 2}} \int_{0}^{1} R^{\beta / 2}(u) Q(u) \mathbf{1}_{R(u)>\varepsilon 2^{k \delta} n^{1 / p}} d u \\
& I_{4}(n, \eta) \ll \sum_{k=1}^{\infty} \frac{n^{(2 p-2-r) / 2 p}}{2^{k \delta(2+r) / 2}} \int_{0}^{1} R^{r / 2}(u) Q(u) \mathbf{1}_{R(u) \leq \varepsilon 2^{k \delta} n^{1 / p}} d u .
\end{aligned}
$$

Clearly $I_{1}(n, \eta) \ll \eta^{(r-p) / p}$ and consequently

$$
\lim _{\eta \rightarrow 0} \limsup _{n \rightarrow \infty} I_{1}(n, \eta)=0 .
$$


Now

$$
I_{2}(n, \eta) \ll n^{(p-1) / p} \int_{0}^{1} Q(u) \mathbf{1}_{R(u)>\varepsilon n^{1 / p}} d u,
$$

and it follows from (3.16) that

$$
\lim _{n \rightarrow \infty} I_{2}(n, \eta)=0
$$

In the same way

$$
I_{3}(n, \eta) \ll n^{(2 p-2-\beta) / 2 p} \int_{0}^{1} R^{\beta / 2}(u) Q(u) \mathbf{1}_{R(u)>\varepsilon n^{1 / p}} d u .
$$

Applying Lemma 6.2 with $b=\beta / 2$, it follows from (6.13) that

$$
\lim _{n \rightarrow \infty} I_{3}(n, \eta)=0 \text {. }
$$

To control $I_{4}(n, \eta)$, we first note that, by Lemma 6.2 with $c=r / 2$,

$$
\frac{n^{(2 p-2-r) / 2 p}}{2^{k \delta(2+r) / 2}} \int_{0}^{1} R^{r / 2}(u) Q(u) \mathbf{1}_{R(u) \leq \varepsilon 2^{k \delta} n^{1 / p}} d u \ll \frac{1}{2^{k p \delta}},
$$

and

$$
\lim _{n \rightarrow \infty} \frac{n^{(2 p-2-r) / 2 p}}{2^{k \delta(2+r) / 2}} \int_{0}^{1} R^{r / 2}(u) Q(u) \mathbf{1}_{R(u) \leq \varepsilon 2^{k \delta} n^{1 / p}} d u=0 .
$$

Hence, by the dominated convergence theorem,

$$
\lim _{n \rightarrow \infty} I_{4}(n, \eta)=0
$$

Finally, the tightness follows from (6.9), (6.10), (6.11), (6.12), (6.14) and (6.15). This completes the proof of Theorem 3.3.

\section{References}

[1] Z. Ciesielski (1960), On the isomorphism of the spaces $\mathcal{H}_{\alpha}$ and $m$, Bull. Acad. Pol. Sci. 7 $217-222$.

[2] J. Dedecker, S. Gouëzel and F. Merlevède (2010), Some almost sure results for unbounded functions of intermittent maps and their associated Markov chains. Ann. Inst. Henri Poincaré Probab. Stat. 46 796-821.

[3] J. Dedecker and F. Merlevède (2007), Convergence rates in the law of large numbers for Banach-valued dependent variables. Teor. Veroyatn. Primen. 52 562-587; translation in Theory Probab. Appl. 52 416-438.

[4] J. Dedecker and F. Merlevède, Moment bounds for dependent sequences in smooth Banach spaces, arXiv:1404.0563 (2014).

[5] J. Dedecker and C. Prieur (2004), Coupling for $\tau$-dependent sequences and applications, $J$. Theor. Probab. 17 861-885.

[6] J. Dedecker and C. Prieur (2005), New dependence coefficients. Examples and applications to statistics, Probab. Theory Related Fields 132 203-236. 
[7] J. Dedecker and E. Rio (2008), On mean central limit theorems for stationary sequences, Ann. Inst. Henri Poincaré Probab. Stat. 44 693-726.

[8] D. Giraudo (2015), Hölderian weak invariance principle for stationary mixing sequences, $J$. Theor. Probab. published online. DOI 10.1007/s10959-015-0633-9

[9] D. Giraudo (2015), Hölderian weak invariance principle under an Hannan type condition, Stochastic Process. Appl. published online. http://dx.doi.org/10.1016/j.spa.2015.09.001

[10] S. Gouëzel (2004), Central limit theorem and stable laws for intermittent maps, Probab. Theory Related Fields 128 82-122.

[11] E. Lesigne and D. Volný (2001), Large deviations for martingales, Stochastic Process. Appl. 96 143-159.

[12] C. Liverani, B. Saussol and S. Vaienti (1999), A probabilistic approach to intermittency, Ergodic Theory Dynam. Systems 19 671-685.

[13] I. Melbourne (2009), Large and moderate deviations for slowly mixing dynamical systems. Proc. Amer. Math. Soc. 137 1735-1741.

[14] F. Merlevède and M. Peligrad (2013), Rosenthal-type inequalities for the maximum of partial sums of stationary processes and examples, Ann. Probab. 41 914-960.

[15] A. Račauskas and C. Suquet (2004), Necessary and sufficient condition for the functional central limit theorem in Hölder spaces, J. Theoret. Probab. 17 221-243.

[16] E. Rio (2000), Théorie asymptotique des processus aléatoires faiblement dépendants, Mathématiques et Applications 31, Springer-Verlag, Berlin.

[17] E. Rio (2013), Inequalities and limit theorems for weakly dependent sequences. 3ème cycle. pp. 170. <cel-00867106>

[18] M. Rosenblatt (1956), A central limit theorem and a strong mixing condition, Proc. Nat. Acad. Sci. U. S. A. 42 43-47.

[19] L. Rüschendorf (1985), The Wasserstein Distance and Approximation Theorems, Z. Wahrsch. Verw. Gebiete 70 117-129. 